\title{
A microscopic perspective of a microreactor
}

\author{
F. Carvalho*, P. Paradiso** and P. Fernandes*
}

*IBB-Institute for Biotechnology and Bioengineering, Centre for Biological and Chemical Engineering, Department of Bioengineering, Instituto Superior Técnico, Universidade Técnica de Lisboa, Lisboa, Portugal **CQE - Centre of Structural Chemistry, Instituto Superior Técnico, Universidade Técnica de Lisboa, Lisboa, Portugal

The use of microreactors in (bio)chemical processes has been gaining relevance in the last decade. The low consumption of reagents, the possibility of continuous operation and the faster translation from lab- to production scale are some of the several advantages of these devices. The whole results in cost reductions in process development [1][2]. Enzyme catalyzed reactions have proved to be an excellent alternative to the use of chemicals due to its ability to catalyze the most complex chemical processes under benign experimental and environmental conditions. In this way, enzymes may be crucial to the implementation of a much more sustainable chemical industry[3][4].

The present work is within such scope, using as model system the immobilization of invertase in glass (silica) microchannels, for the production of fructose syrups through sucrose hydrolysis. The immobilization of the enzyme was achieved through treatment of the substrate with a sequence of coatings (Figure 1). Activation of the inner surface of the microchannels with 3-aminopropyltriethoxysilane (APTES) was followed by the introduction of a spacer, glutaraldehyde. Lastly the enzyme solution was introduced in the presence of sodium cyanoborohydride, in order to enhance the stability of the support-enzyme binding.

The characterization of the coatings at each stage of the immobilization protocol was carried out to confirm the change of the microchannel surface. Such studies were performed using Atomic Force Microscopy (AFM) and Scanning Electron Microscopy (SEM) analysis (Figure 1).

The results obtained, namely the shifts on the surface roughness corroborate that the several coatings were successfully applied and the enzyme immobilized. Moreover, the immobilization approach used proved to be highly effective, resulting on successful continuous use of the microreactor for a period of 30 days with roughly constant full conversion of a sucrose solution of $50 \mathrm{~g} / 1$, at a flow rate of $7 \mu 1 /$ minute (Figure 2 ).

Future work will involve a more extensive characterization of the several coatings by Quartz Crystal Microbalance which will be decisive to achieve a better comprehension of the coating phenomena and hence optimize the immobilization process.

\section{References}

1. Marques M.P.C. and Fernandes P., Molecules, 16:8368-8401, 2011.

2. Fernandes P. et al., Recent Patents on Biotechnology, 5:160-73, 2011.

3. Sheldon R.A., Advanced Synthesis \& Catalysis, 349:1289-1307, 2007.

4. Fernandes P., International Journal of Molecular Sciences, 11:858-879, 2010. 


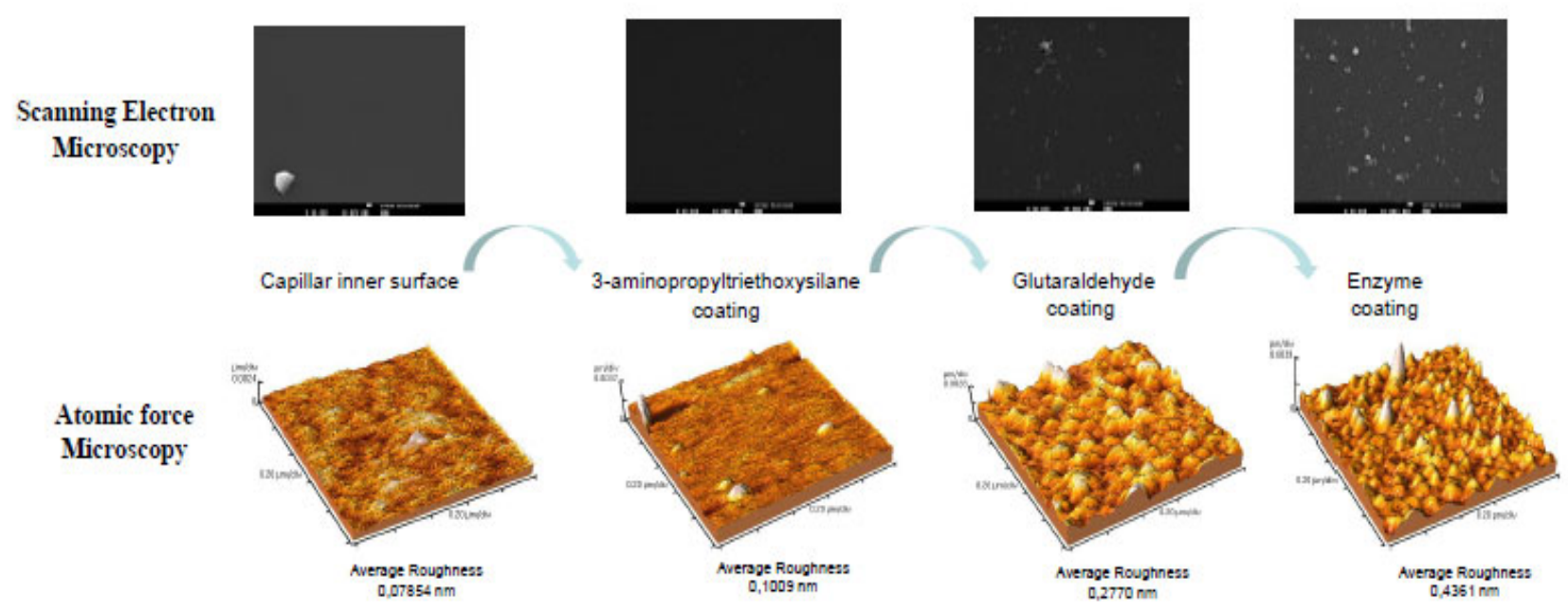

Fig. 1 - Coating sequence and characterization by SEM and AFM.

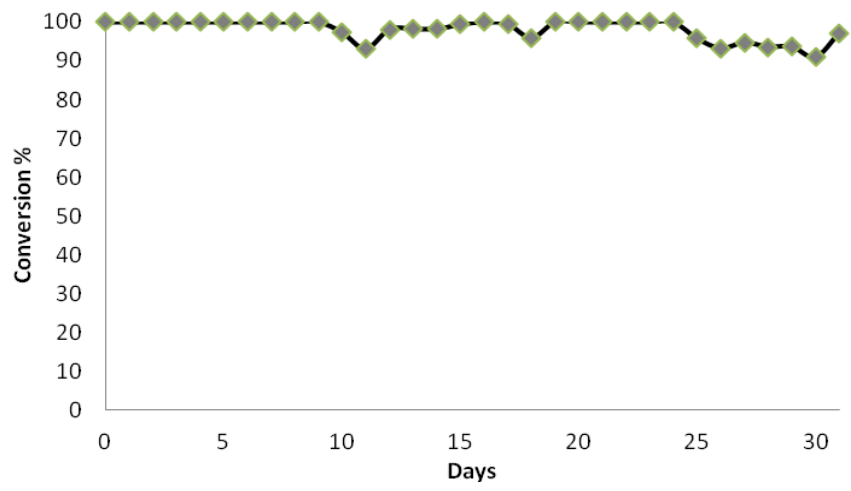

Fig. 2 - Continuous production of fructose syrup through sucrose hydrolysis by immobilized invertase $(50 \mathrm{~g} / 1$ sucrose solution in acetate buffer $0.1 \mathrm{M}, \mathrm{pH} 4.5$; flow rate of $7 \mu 1 /$ minute, $50^{\circ} \mathrm{C}$ ).

The authors would like to thank Fundação para a Ciência e a Tecnologia, Portugal, for financial support through contracts under the program Ciência 2007 awarded to P. Fernandes, for the doctoral grant SFRH/BD/74818/2010 awarded to F. Carvalho and for the doctoral grant SFRH/BD/71990/2010 awarded to Patrizia Paradiso. 